\section{Superior vena cava syndrome associated with Nocardia farcinica infection}

Sofia Abdelkafi, Didier Dubail, Thierry Bosschaerts, Alain Brunet, Guy Van Camp, Michel de Marneffe, Jeanne-Marie De Vaster, Vincent Ninane

\author{
Abstract \\ A case is described of severe Nocardia \\ farcinica infection which mimicked a \\ pulmonary neoplasm with pneumonia, \\ superior vena cava syndrome, peri- \\ carditis, and hypertrophic osteo- \\ arthropathy. Treatment with trimetho- \\ prim-sulphamethoxazole and surgery res- \\ ulted in complete recovery. \\ (Thorax 1997;52:492-493)
}

Keywords: Nocardia farcinica, superior vena cava syndrome, pericarditis, hypertrophic osteoarthropathy.

A 41 year old male smoker and alcoholic presented with a three month history of cough. $\mathrm{He}$ also complained of night sweats, burning pain in the lower legs, and a $7 \mathrm{~kg}$ weight loss. Chest radiography performed three weeks earlier in another hospital had revealed a right upper lobe pneumonia which failed to respond to amoxycillin. The patient was working as a cook in a hospital.

Physical examination only revealed inspiratory crackles over the right upper lobe. Finger clubbing was not present. The chest radiograph was unchanged. A tuberculin skin test was negative. Fibreoptic bronchoscopy was normal and extensive search for infectious organisms including Mycobacterium tuberculosis in the sputum and in bronchoalveolar lavage fluid from the right upper lobe was negative.

Ten days later distended neck veins were noted on clinical examination and a repeated chest radiograph revealed widening of the mediastinum and an enlarged heart (fig 1). A CT scan of the chest showed consolidation of the right upper lobe without cavitation, multiple enlarged mediastinal lymph nodes with compression of the superior vena cava, and a voluminous pericardial effusion. Compression of the superior vena cava was confirmed by angiography. Hypertrophic osteoarthropathy was suggested by radionuclide bone scanning and radiography of the lower legs confirmed the presence of subperiosteal new bone formation (fig 2).

A mediastinoscopy was performed and revealed a purulent mediastinitis; pericardial drainage was also performed and showed a yellow fluid. In both cases direct examination of smears revealed Gram positive organisms. After culture these microorganisms formed aer- ial hyphae that were identified as Nocardia farcinica by the Division of Bacterial and $\mathrm{My}$ cotic Diseases, Centers for Disease Control (Atlanta, Georgia, USA). Positive biochemical tests which allowed differentiation from $\mathrm{No}$ cardia asteroides included growth at $45^{\circ} \mathrm{C}$ for three days, hydrolysis of acetamide, and resistance to cefamandole and tobramycin in vitro. ${ }^{12}$ The strain was sensitive to amikacin and imipenem and moderately susceptible to sulphamethoxazole/trimethoprim (minimum inhibitory concentration $38 / 2 \mu \mathrm{g} / \mathrm{ml}$ ). Co-trimoxazole (trimethoprim $1600 \mathrm{mg}$ and sulphamethoxazole $8000 \mathrm{mg}$ ) four times daily was instituted and mediastinal and pericardial drainage were continued for five days. The patient's condition improved quickly. A CT scan of the thorax showed a major resolution of the mediastinal widening and complete resolution of the pericardial effusion. Two weeks

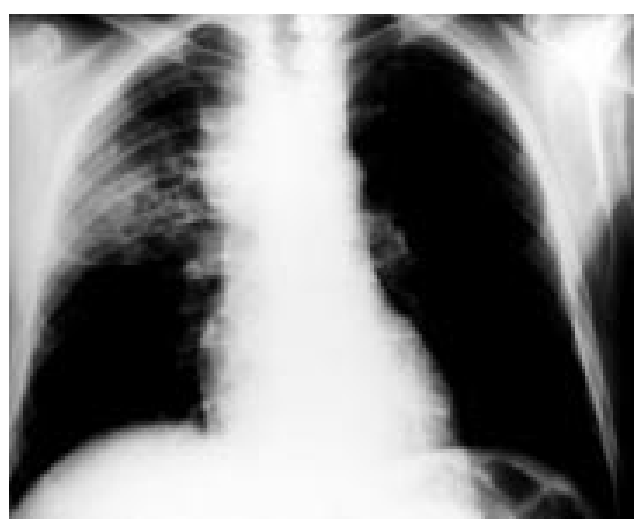

Figure 1 Posteroanterior chest radiography of the thorax showing a right upper lobe pneumonia and widening of the mediastinum.

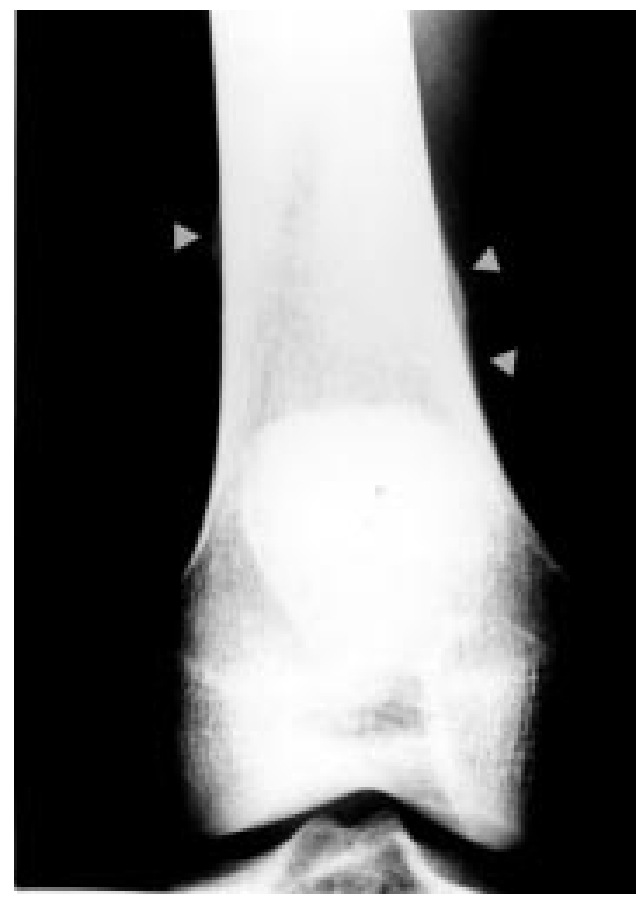

Figure 2 Hypertrophic pulmonary osteoarthropathy: the proliferative periostitis (arrowheads) is seen as a dense irregular line separated from the cortex of the femur by a narrow band of radiolucency.
10 July 1996

Accepted for publication

2 August 1996 
after the surgical procedure the patient was discharged with the same drug regimen. At that time he appeared well and the distended neck veins had disappeared.

Two weeks later distension of the jugular veins had recurred and clinical examination also revealed oedema in the ankles and lower legs. No widening of the mediastinum and no enlargement of the heart was present on the chest radiograph. Echocardiography was suggestive of a constrictive pericarditis that was confirmed by right and left heart haemodynamic measurements. Surgical removal of the constrictive pericardium was performed. On histological examination the pericardium was thickened and showed fibrous tissue. Culture for Nocardia remained negative. Recovery was then uneventful. The antibiotic was well tolerated and was discontinued after three months, at which time the CT scan of the thorax was normal and the hypertrophic pulmonary osteoarthropathy had resolved. The patient remained asymptomatic at follow up two years later.

Apart from alcoholism, no other congenital or acquired disorder of immunity was found. In particular, quantitative assessment of immunoglobulins and their subclasses, as well as serum protein electrophoresis, were normal and there was no complement disorder. Delayed hypersensitivity was also established by skin tests and quantification of $\mathrm{T}$ cells was normal. Measurement of antibodies against HIV was negative. In addition, there was no clinical feature of alcoholic cirrhosis and laboratory assessment after resolution of the sepsis showed no abnormality to indicate cirrhosis.

\section{Discussion}

This case report of severe Nocardia farcinica infection had clinical and radiological features strongly suggestive of lung cancer. Indeed, the patient presented with lobar infiltrates complicated by mediastinal widening, superior vena cava syndrome, and hypertrophic pulmonary osteoarthropathy. Bronchoscopic examination, however, did not show any malignant disease and infection was confirmed by mediastinoscopy. Acute Nocardia farcinica pericarditis was also present and ultimately was complicated by constrictive pericarditis.

Nocardia asteroides is the predominant source of human infection due to Nocardia species. Its dissemination can occur in almost every organ, and superior vena cava syndrome ${ }^{3}$ with acute purulent pericarditis and constrictive pericarditis $^{4}$ have occasionally been reported. Nocardia farcinica has also recently been recognised as a potential human pathogen ${ }^{56}$ and cases of human infection are increasingly identified. Among the different clinical types of infection the most frequent include pulmonary, ${ }^{56}$ cutaneous, ${ }^{67}$ and cerebral presentations. ${ }^{68}$ We believe that hypertrophic osteoarthropathy in this patient was related to the intrathoracic sepsis. ${ }^{9}$ Indeed, hypertrophic osteoarthropathy was originally reported with chronic pleuropulmonary sepsis including empyema, bronchiectasis, and lung abscess. In addition, the fact that hypertrophic osteoarthropathy resolved after complete recovery also supports the view that the infection was causative. Although the precise pathophysiology of hypertrophic osteoarthropathy remains largely unknown, ${ }^{9}$ malignant chest neoplasms account for most cases and, here also, treatment of the causative tumour may lead to resolution of hypertrophic osteoarthropathy.

The severity of the clinical presentation in our case supports the view that Nocardia farcinica is often associated with life threatening systemic infection. ${ }^{7}$ This is particularly likely to be the case in patients with an underlying immunosuppressive condition ${ }^{78}$ such as chronic alcoholism, as was encountered in our patient. Identification of this particular pathogen and differentiation from Nocardia asteroides is of clinical importance since Nocardia farcinica characteristically shows resistance to numerous antimicrobial agents ${ }^{1}$ and is probably associated with a higher pathogenicity. ${ }^{10}$ Nocardia farcinica isolates are almost always susceptible to imipenem, ciprofloxacin, amikacin, and sulphamethoxazole. Sulphonamides (with or without trimethoprim) appear to be the treatment of choice for Nocardia farcinica as they are for other Nocardia species. ${ }^{2}$ The present isolate, however, was moderately susceptible to sulphamethoxazole. At the time this information was available there was already a major clinical improvement such that co-trimoxazole was continued. We also believe that mediastinal and pericardial drainage, as well as pericardiectomy, played an important part in the favourable outcome of our patient. In fact, surgical interventions are required in some patients with Nocardia infection, particularly in those with Nocardia asteroides pericarditis. ${ }^{3}$

This case further emphasises the fact that Nocardia farcinica is associated with life threatening infection and must be differentiated from Nocardia asteroides since it is resistant to numerous antibiotics. This infectious disorder must also be considered in patients who present with superior vena cava syndrome.

The authors acknowledge the assistance in identification of the Nocardia farcinica of Dr June M Brown and Dr Michael M McNeil from the Division of Bacterial and Mycotic Diseases, National Center for Infectious Diseases, USA.

1 Wallace RJ, Tsukamura M, Brown BA, Brown J, Steingrube VA, Zhang YS, et al. Cefotaxime-resistant Nocardia as teroides strains are isolates of the controversial species Nocardia farcinica. 7 Clin Microbiol 1990;28:2726-32.

$2 \mathrm{McNeil}$ MM, Brown JM. The medically important aerobic Actinomycetes: epidemiology and microbiology. Clin Microbiol Rev 1994;7:357-417.

3 Poland GA, Jorgensen CR, Sarosi GA. Nocardia asteroides pericarditis: report of a case and review of the literature. Mayo Clin Proc 1990;65:819-24.

4 Pitchenik AE, Zaunbrecher AE. Superior vena cava syndrome caused by Nocardia asteroides. Am Rev Respir Dis 1978;117:795-8.

5 Tsukamura M, Ohta M. Nocardia farcinica as a pathogen of lung infection. Microbiol Immunol 1980;24:237-41.

6 Holm P. Seven cases of human nocardiosis caused by $\mathrm{No}$ cardia farcinica. Sabouraudia 1975;13:161-9.

7 Schiff TA, Mc Neil MM, Brown JM. Cutaneous Nocardia farcinica infection in a non-immunocompromised patient: farcinica infection in a non-immunocompromised patient:

8 Miksits K, Stoltenburg G, Neumayer HH, Spiegel H, Schaal $\mathrm{KP}$, Cervos-Navarro J, et al. Disseminated infection of the central nervous system caused by Nocardia farcinica. the central nervous system caused by Nocardia farcinica.

9 Finger clubbing and hypertrophic pulmonary osteoarthropathy. BMF 1977;2:785

10 Desmond EP, Flores M. Mouse pathogenicity studies of Nocardia asteroides complex species and clinical correlation with human isolates. FEMS Microbiol Lett 1993;110:281- 